\title{
Addressing COVID-19 Spread: Development of Reliable Testing System for Mask Reuse
}

\author{
Haoxian Lu ${ }^{1}$, Dawen Yao ${ }^{1}$, Joanne Yip ${ }^{2}$, Chi-Wai Kan ${ }^{2}$, Hai Guo ${ }^{1 *}$ \\ ${ }^{1}$ Air Quality Studies, Department of Civil and Environmental Engineering, The Hong Kong Polytechnic University, Hong \\ Kong, China \\ ${ }^{2}$ Institute of Textiles and Clothing, The Hong Kong Polytechnic University, Hong Kong, China
}

\begin{abstract}
While the novel coronavirus pandemic (COVID-19) continues to wreak havoc globally, self-protection from possible infection by wearing a mask in daily life has become the norm in many places. The unprecedented demand for masks has now attracted attention on their filtration efficiency. Furthermore, the widespread use of disposable masks has led to shortage of filter materials and problems with their haphazard disposal. In this study, a testing system that is based on standardized methods has been established and enhanced to reliably measure the particle filtration efficiency (PFE) of masks. Quality control experiments that examine the filtration efficiency of polystyrene latex (PSL) particles that are $0.1 \mu \mathrm{m}$ in size and sodium chloride $(\mathrm{NaCl})$ particles that range from $0.01-1.0 \mu \mathrm{m}$ are conducted to determine the reliability of the testing system. Moreover, various textile materials are tested to fabricate 3-layer face masks, and the PFE of these masks is tested by using the proposed testing system to find the most suitable materials and the likelihood of their reusability. Among the tested materials, polytetrafluoroethylene (PTFE) used as the membrane in the filter layer has the highest PFE of $88.33 \% \pm 1.80 \%$, which is mainly due to its dense and multilayer structure. The air permeability of the self-developed masks ranges from 1.41 \pm 0.04 to $1.93 \pm 0.08$, less breathable than the commercial masks. The reusability of a mask that uses PTFE as the membrane in the filter layer is tested by gently washing the mask 30 times and then drying the mask in air before the PFE is measured. The PFE is only reduced by 10-20\% after 30 washes, thus indicating the potential reusability of the mask. The findings in this study will contribute to reducing the pressure of mask shortages and are an environmentally friendly solution to the massive use of disposable masks.
\end{abstract}

Keywords: COVID-19; Mask testing system; Reusable mask; Particle filtration efficiency.

\section{INTRODUCTION}

The new coronavirus (COVID-19) has become a global public health concern because it is highly contagious with severe or even fatal health consequences. With the rapid spread of COVID-19, self-protection has become crucial to avoid contracting this disease. As a highly infectious disease, the two main routes of transmission include direct transmission (e.g., spraying droplets emitted from aerosolized particles through sneezing, coughing or talking), and indirect transmission (contact with surfaces that contain the virus or objects that have been in contact with an infected individual) (Liu, 2020; Ningthoujam, 2020; Tomar and Gupta, 2020). However, the former has been documented to be a more prevalent form of transmission, so an effective mask must be worn to obstruct the transmission of the virus and block

\footnotetext{
* Corresponding author.

E-mail address: ceguohai@polyu.edu.hk
}

its spread when conducting daily life activities (Bałazy et al., 2006; MacIntyre and Chughtai, 2020). This has led to the unprecedented demand and shortage of masks, along with the problem of ineffective masks. On the one hand, there are a variety of different masks on the market. Although most commercially available masks claim effectiveness against contact with airborne particles, their actual performance has not been tested adequately. For example, Cheng (2020) reported that there was no quality control in face mask production in Pakistan. In Italy, in order to meet the huge demand of face masks, some industries have reset the production chain, shifting from the usual target products to the production of masks. However, the quality of the masks is questionable and needs to be tested (Amendola et al., 2020). In addition, Lam et al. (2020) examined 160 mask brands on the market and found that $48.8 \%$ of them were substandard and/or invalid. With COVID-19 as an urgent public health issue now, it has become imperative to test and validate the performance of these masks with reliable testing equipment. On the other hand, COVID-19 has led to the unprecedented demand for masks, which has resulted in a 
shortage of materials to fabricate them. Moreover, most of the masks currently found on the market are disposable (i.e., surgical, N95 and KF94 masks). The widespread use of such disposable masks is having a detrimental effect on the environment, as additional resources and human resources are needed to properly dispose of used masks which might otherwise end up in the oceans or landfills. As such, it is timely to develop effective yet reusable masks, not only to address the needs of the current epidemic but also to conserve resources.

One of the key parameters of masks is their particle filtration efficiency (PFE) which reflects the ability of masks and/or filters to block the inhalation of different types of particles. Basically, PFE testing follows the methods stipulated in various standards, which include ASTM F2299 (the United States) and YY 0469-2011/GB19083-2010 (Chinese), and the standard provided by the National Institute for Occupational Safety and Health (NIOSH). In ASTM F2299, polystyrene latex (PSL) particles of a specific size are used to evaluate the PFE of a material. Although the applicable particle size range is from $0.1 \mu \mathrm{m}$ to $5.0 \mu \mathrm{m}$, PSL particles of $0.1 \mu \mathrm{m}$ are generally used for testing as it is the most penetrating particle size. In YY 0469-2011, sodium chloride $(\mathrm{NaCl})$ particles that range from $0.01 \mu \mathrm{m}$ to $1.0 \mu \mathrm{m}$ are used as the index in PFE testing of masks, while the NIOSH standard is used to test masks to filter $\mathrm{NaCl}$ particles that are $0.3 \mu \mathrm{m}$ in size. Although these standard methods provide basic instructions and parameters for PFE testing, they do not offer specific settings for testing systems and details of the equipment involved. Moreover, some of the standard methods have limitations. For instance, a device that is recommended for measuring particle concentration (an optical particle counter (OPC)) is low in accuracy and size-resolution (Rengasamy et al., 2011). Moreover, the standards do not strictly stipulate the size of the particles to be tested, thus resulting in differences and uncertainties in the results with the different testing agents and systems. Lastly, the impacts of different types of particles on the testing results have been hardly considered and discussed. Apart from the PFE, air permeability is another important parameter for a mask. The air permeability indicates how comfortable the mask is to breathe. The more permeable a mask is, the lower air resistance it has and the more comfortable it is to wear. In general, there is a balance between air permeability and filtration efficiency. A satisfactory mask should own a good ability of filtration with an acceptable air permeability (Konda et al., 2020).

Therefore, this study aims to establish a testing system that examines two types of particles to accurately measure the PFE of various face masks. The proven testing system is subsequently used to determine suitable fabrics for reusable masks. The materials for the most effective reusable face mask are presented in this study. Finally, the air permeability of the masks is measured to indicate the wearing comfort of the masks.

\section{METHODS AND MATERIALS}

\section{PFE Testing System Setup}

According to standard methods, the established testing system has to incorporate a particle generation unit, a sample holding unit and a detection unit. A detailed schematic of the testing system is shown in Fig. 1.

The particle generation unit is an atomizer (Model 7.811, Grimm, Germany), which was used to generate the PSL and $\mathrm{NaCl}$ particles and provide sufficient air flow in the testing system by supplying particle-free dilute gas. By adding 2-3 drops of PSL solution (Thermo Scientific, USA) into $8 \mathrm{~mL}$ of Milli-Q water, an aqueous solution of PSL particles was produced for atomization, which generated the PSL particles. To generate the $\mathrm{NaCl}$ particles, $25 \mathrm{~mL}$ of $0.025 \mathrm{~g} \mathrm{~mL}^{-1} \mathrm{NaCl}$ solution was added into the atomizer. The count median diameter (CMD) of the generated PSL particles was $101.2 \mathrm{~nm}$ with the geometric standard deviation (GSD) of $4.6 \mathrm{~nm}$, while the $\mathrm{CMD}$ of the generated $\mathrm{NaCl}$ particles was $42.8 \mathrm{~nm}$

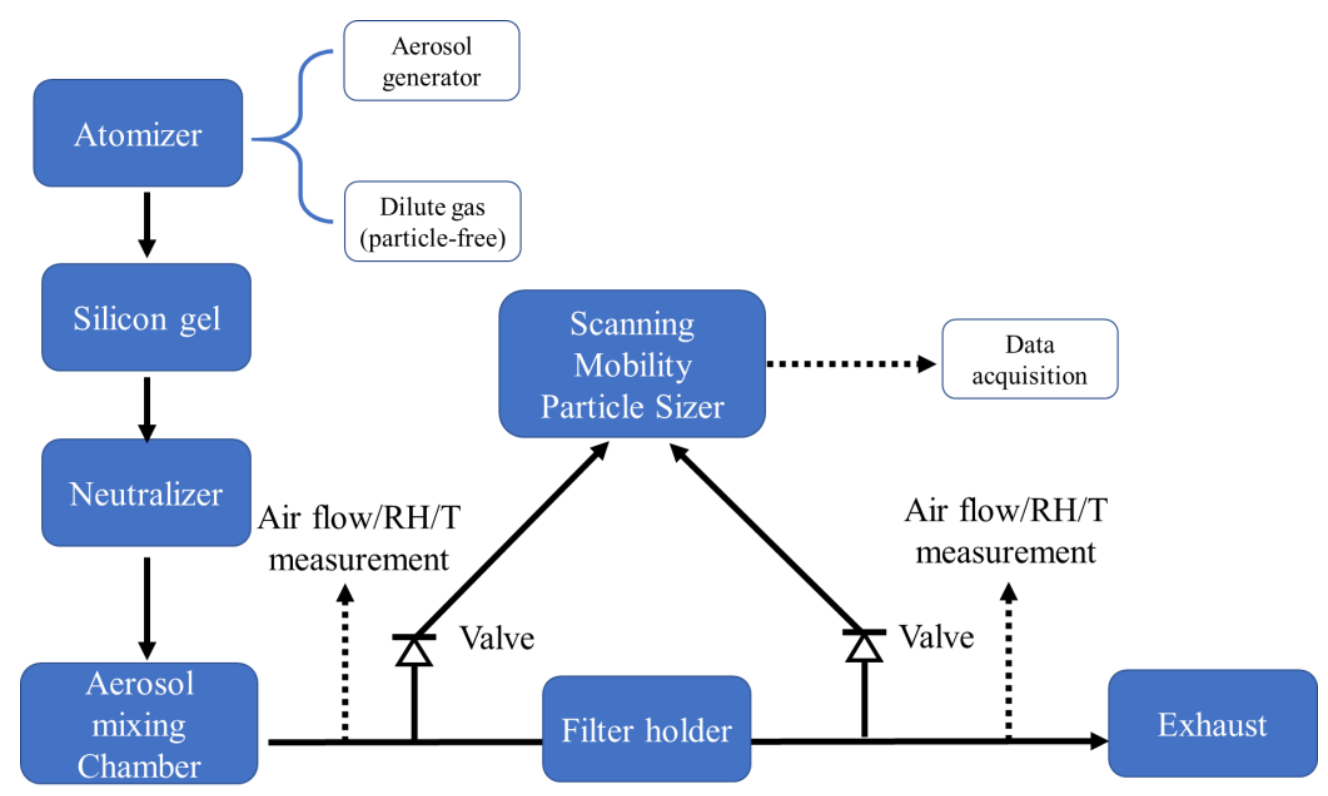

Fig. 1. Schematic diagram of the PFE testing system. 
with the GSD of $0.9 \mathrm{~nm}$. The generated particles were introduced into an aerosol neutralizer (TSI Incorporated, Model 3077A) to eliminate the electrostatic charges and ensure stability of the particle surface charge. Silica gel was used to remove the water droplets and water vapour produced from the atomizer and maintain a constantly low humidity in the system. The particles were then mixed in a mixing chamber (cuboid shaped with a volume of $\sim 2.5 \mathrm{~L}$ ) before they were passed through a mask placed in the sample holder. The air flow, relative humidity (RH) and temperature (T) were measured in the upstream and downstream of the sample holder, respectively. The sample holder is a customized accessory in which a mask with a cross-sectional area of $\sim 14 \mathrm{~cm}^{2}$ can be properly placed, and the holder is sealed with a layer of rubber to prevent air leakage. The detection unit is a scanning mobility particle sizer (SMPS) (Model 5.400, Grimm, Germany) which can measure the number concentration of particles in the upstream and downstream of the sample holder, respectively. The SMPS is a highly sensitive and accurate instrument which electronically measures the size-resolved number concentration of particles with a maximum time-resolution of 5 seconds for each size bin. During testing, the number concentration of particles was acquired at an interval of 10 seconds for a specific size of PSL particles. As for the $\mathrm{NaCl}$ particles, particles that ranged from $0.01 \mu \mathrm{m}$ to $1.0 \mu \mathrm{m}$ were categorized into 44 size bins and measured at a time interval of 7 mins. The differences in particle concentration of each particle size between the upstream and downstream of the sample holder were used to calculate the PFE values $(\mathrm{PFE}=100 \% \times(1-$ average downstream concentration/average upstream concentration)). Each test was about 10-30 minutes to obtain a stable PFE value. Exhaust gas was discharged after all of the generated particles were removed by using a high-efficiency particulate air (HEPA) filter. Compared with the methods recommended in the NIOSH and ASTM F2299 standards, different types of particles were generated for the examination of the PFE of masks in the study. This study also adopted a more advanced and accurate instrument, i.e., SMPS. Besides, silica gel was used to remove the moisture generated by the atomizer, thereby protecting the instrument and eliminating the influence of moisture on the PFE results. Moreover, the duration of the PFE test was longer than previous methods to obtain accurate results (10-30 minutes vs. 1-5 minutes) (Rengasamy et al., 2017).

\section{Operational Conditions of Testing System}

The PFE results are influenced by several factors, including humidity, temperature, air velocity, and aerosol generation (Rengasamy et al., 2017; Yang et al., 2007). Thus, the operational conditions of the testing system were controlled. PFE testing was conducted under a controlled temperature $\left(20-25^{\circ} \mathrm{C}\right)$ and relative humidity $(40 \%-55 \%)$ with the testing system. Previous study indicated that temperature and relative humidity in these ranges had no remarkable effects on the PFE results (Yang et al., 2007). The air flow in the testing system was 7.3-7.5 $\mathrm{L} \mathrm{min}^{-1}$, which was consistent for all of the tests. The setting of air flow was referred to the ASTM F2299 standard. The airtightness of the system was determined by the consistency of the upstream and downstream air flows. The air velocity in the sample holder was $10 \mathrm{~cm} \mathrm{~s}^{-1}$, which meets the requirement and/or suggestion in the standard methods (i.e., $0.5-25 \mathrm{~cm} \mathrm{~s}^{-1}$ ). It has been proved that air velocity ranging from $4 \mathrm{~cm} \mathrm{~s}^{-1}$ to $16 \mathrm{~cm} \mathrm{~s}^{-1}$ has no discernible effects on the PFE results (Sachinidou et al., 2017). The initial range of number concentration of particles produced by the atomizer was $10^{2}-10^{3}$ counts $\mathrm{cm}^{-3}$, following the suggestion in the standard.

\section{Air Permeability Test}

Air permeability was measured using KES-F8 Air Permeability Instrument (API) (Kato Tech Co., Ltd, Kyoto, Japan). Values were obtained with minute amounts of ventilation (i.e., $4 \mathrm{cc} \mathrm{cm}^{-2} \mathrm{sec}^{-1}$ ). Both front and back sides of each sample were measured for three times, respectively. Totally, six values were derived and averaged to represent the air permeability of a mask.

\section{Materials for Testing}

Seven mask samples were tested, including three types of commercial masks (i.e., KF94, N95 and surgical masks) to validate the testing system and four potentially reusable masks developed in this study. The samples are shown in Figs. 2(A)-2(G). Samples A-D are potentially reusable masks while Samples E-G are the commercial masks. Sample A has two layers, namely $100 \%$ woven cotton fabric (W100, pore size: $14.6-41.6 \mu \mathrm{m})$ as the outer layer, and $92 \%$ cotton $/ 8 \%$ lycra knitted fabric (J92, pore size: $24.6-44.3 \mu \mathrm{m}$ ) as the inner layer. W100 and J92 are commonly used fabrics for clothing. W100 underwent a water repellent and antimicrobial treatment. Samples B-D are three-layer masks. Sample B is $40 \mathrm{~g} \mathrm{~m}^{-2}$ (gsm) of non-woven $100 \%$ polypropylene (PP) fabric with a pore size of $6.5-72.7 \mu \mathrm{m}$ and used as the filter layer between W100 and J92, while Sample C is 20 gsm of PP fabric and used as the membrane for the filter layer. In addition, Sample D is 30 gsm of $100 \%$ polytetrafluoroethylene (PTFE, pore size: $0.2-3.0 \mu \mathrm{m}$ ) and used as the membrane for the filter layer between $\mathrm{W} 100$ and J92. The reusability of Samples A-D was tested by immersing them into deionized (DI) water at $100^{\circ} \mathrm{C}$ for 30 minutes, then rinsed with DI water at room temperature and dried in air. Both the washed and unwashed samples (Samples A-D) were tested for PFE by using the testing system.

\section{RESULTS AND DISCUSSION}

\section{System Validation}

Figs. 3(a) to 3(c) shows the PFE results of the N95, KF94 and surgical masks which are obtained by using the testing system to remove $0.01 \mu \mathrm{m}-1.0 \mu \mathrm{m} \mathrm{NaCl}$ particles based on the YY 0469-2011. Both the N95 and KF94 masks have high PFE against particles that are $0.01 \mu \mathrm{m}-1.0 \mu \mathrm{m}$ in size, and the lowest PFE of $95 \%$ and $94 \%$ against particles that are approximately $0.04 \mu \mathrm{m}$ with the N95 and KF94 masks, respectively, thus indicating their excellent filtration effectiveness. The PFE of the surgical mask, however, is slightly lower, with the lowest PFE of about $83 \%$ against particles that are $0.03 \mu \mathrm{m}-0.1 \mu \mathrm{m}$ in size. Table 1 presents 


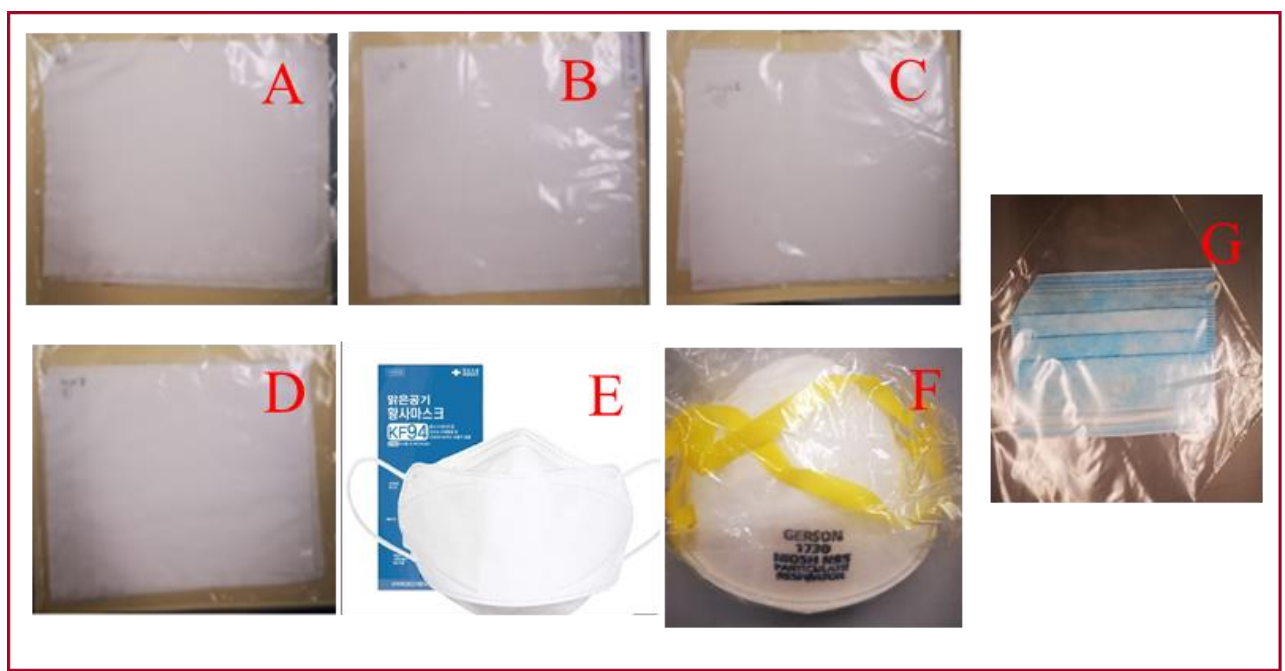

Fig. 2. Mask samples tested in study: (A-D) materials for reusable masks, and (E) KF94, (F) N95 and (G) surgical masks.
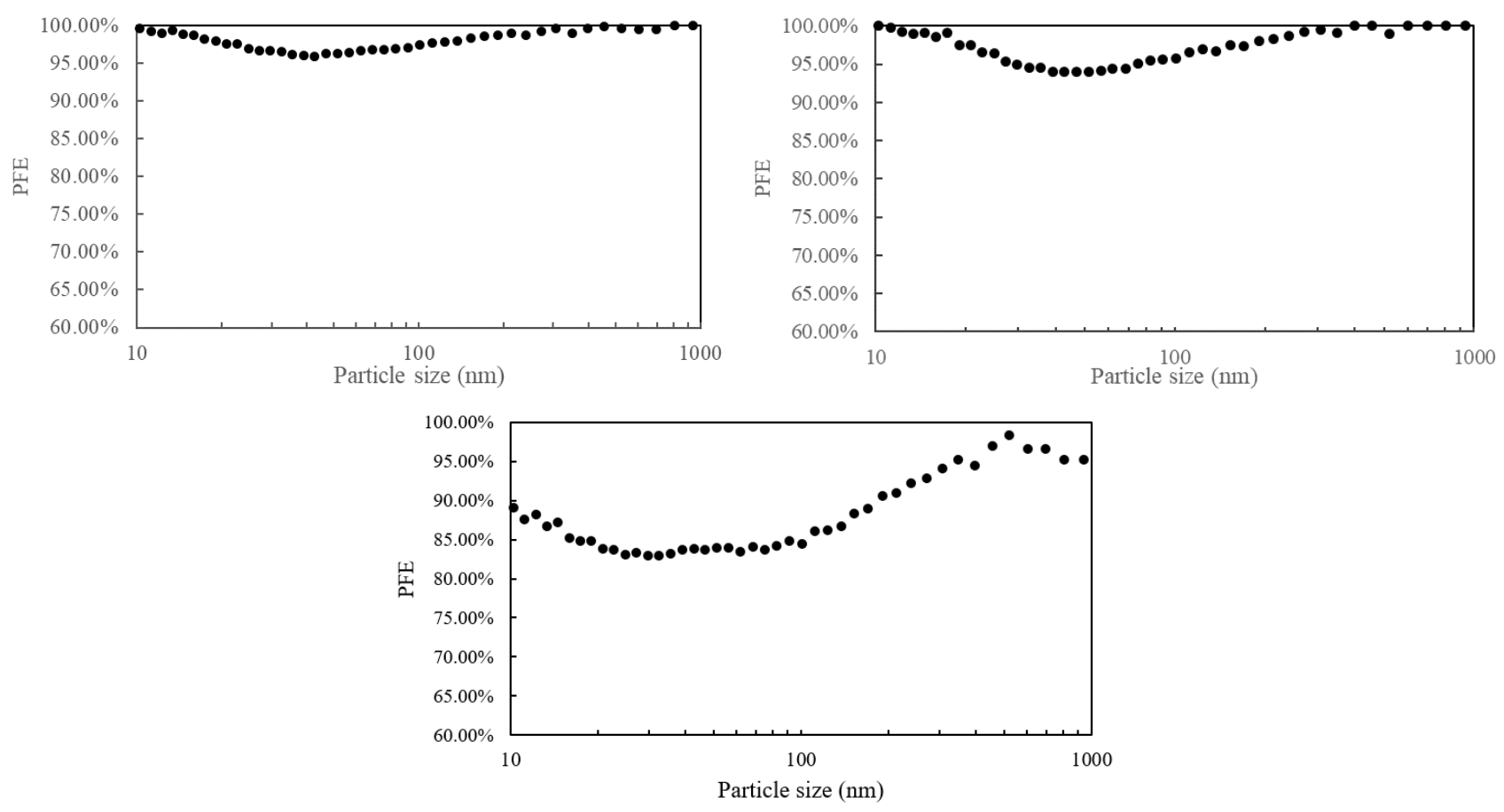

Fig. 3. PFE of (a) N95 mask; (b) KF94 mask; and (c) surgical mask against NaCl particles that range from $0.01 \mu \mathrm{m}$ to $1.0 \mu \mathrm{m}$.

Table 1. PFE of N95, KF94 and surgical masks against $0.1 \mu \mathrm{m}$ PSL particles.

\begin{tabular}{lll}
\hline Sample & PFE & $\begin{array}{l}\text { Mean } \pm \text { Standard } \\
\text { deviation }\end{array}$ \\
\hline N95 (Test 1) & $95.1 \%$ & $93.8 \% \pm 1.2 \%$ \\
N95 (Test 2) & $92.9 \%$ & \\
N95 (Test 3) & $93.3 \%$ & \\
KF94 (Test 1) & $96.7 \%$ & $96.6 \% \pm 1.2 \%$ \\
KF94 (Test 2) & $97.7 \%$ & \\
KF94 (Test 3) & $95.4 \%$ & \\
Surgical mask (Test 1) & $83.2 \%$ & $80.2 \% \pm 7.6 \%$ \\
Surgical mask (Test 2) & $85.8 \%$ & \\
Surgical mask (Test 3) & $71.6 \%$ & \\
\hline
\end{tabular}

the PFE results of the N95, KF94 and surgical masks against PSL particles that are $0.1 \mu \mathrm{m}$ in size in accordance with ASTM F2299. The PFE of the N95, KF94 and surgical masks are $93.8 \% \pm 1.2 \%, 96.6 \% \pm 1.2 \%$ and $80.2 \% \pm 7.6 \%$, respectively. The PFE results of the tested masks were calculated using the upstream and downstream concentrations of only $0.1 \mu \mathrm{m}$ particles measured by the SMPS regardless of particle types (PSL or $\mathrm{NaCl}$ ). The bigger variation in the PFE results of surgical masks might be attributed to the use of different surgical mask samples for testing. Overall, the results are in line with the $\mathrm{PFE}$ against $\mathrm{NaCl}$ particles that are $0.1 \mu \mathrm{m}$ in size (all $p>0.05$ ) (i.e., $97.4 \%, 95.7 \%$ and $84.4 \%$ for the N95, KF94 and surgical masks, respectively), thus suggesting the independence of the PFE values from the 
type of particle. Overall, the N95 and KF94 masks both show an excellent performance in blocking ultrafine particles with a PFE of over 94\%, which holds true for both the PSL particles that are $0.1 \mu \mathrm{m}$ in size or the $\mathrm{NaCl}$ particles that range from 0.01 to $1.0 \mu \mathrm{m}$. Compared to the N95 and KF94 masks, the surgical mask offers a lower PFE against $\mathrm{NaCl}$ particles that range from $0.01-1.0 \mu \mathrm{m}$ in size and PSL particles that are $0.1 \mu \mathrm{m}$. Nevertheless, the filtration efficiency of the surgical mask is still outstanding (all PFE > 80\%). The comparable PFE of the N95, KF94 and surgical masks measured in the study with those of previous studies (> 95\% and $>94 \%$ PFE for N95 and KF94 masks, respectively, and 55-88\% PFE for surgical masks) confirm that our testing system is reliable (Liu and Hsu, 2003; Rengasamy et al., 2011; Rengasamy et al., 2017; Wang and Tronville, 2014).

As mentioned earlier, the OPC has some drawbacks which may cause fluctuations in the PFE results. That is, the OPC not only detects the PSL or $\mathrm{NaCl}$ particles but also water droplets that are generated from the atomizer. Hence, the number concentration of the particles upstream could be overestimated by unintentionally including the water droplets (Kohli and Mittal, 2011), while there are no water droplets in the number concentration of the particles downstream as the mask should adsorb the water. Consequently, the PFE could be overestimated. Secondly, the OPC measures the number concentration of particles of a specific size range instead of a specific size. In fact, when the size distribution of particles generated by using $0.1 \mu \mathrm{m}$ of a PSL standard solution was examined by the SMPS, it was found that in addition to mostly $0.1 \mu \mathrm{m}$ particles (Fig. S1), particles with different sizes were also generated due to coagulation (Yasui and Higashitani, 1988; Fukasawa and Adachi, 2010). Thus, when testing mask samples with a solution that contains $0.1 \mu \mathrm{m}$ PSL, the concentration of particles aside from those that are $0.1 \mu \mathrm{m}$ in size are also counted by the OPC, thus leading to inaccurate PFE results. The SMPS used in this study can overcome these disadvantages because it is capable of measuring the number concentration of particles of a specific size from $0.005 \mu \mathrm{m}$ to $1.0 \mu \mathrm{m}$ based on the electrical mobility of the particles, and does not consider water droplets as aerosol particles (e.g., $0.1 \mu \mathrm{m}$ PSL). Butanol-saturated air was drawn into a condensing chamber, where supersaturated butanol vapor condensed on the surface of the particles rather than water droplets. In fact, silica gel was used to remove the water droplets and water vapour produced from the atomizer before particles entered the SMPS (Fig. 1) because if the water droplet gets into the instrument, it will affect the charges on particles, size screening ability of DMA and counting ability of CPC.

\section{Fabrics for Masks with High PFE}

Table 2 shows the test results of the four developed fabric masks for PFE against PSL particles that are $0.1 \mu \mathrm{m}$ in size. Sample D has the highest PFE, with an average value of $88.3 \% \pm 1.8 \%(p<0.1)$, followed by Samples B and C which have a PFE of $51.6 \% \pm 5.1 \%$ and $37.4 \% \pm 0.1 \%$, respectively. Sample A has the lowest PFE of $22.9 \% \pm 6.0 \%$ $(p<0.1)$. The discrepancies in the PFE are caused by several factors. First, the extremely low PFE of Sample A is due to
Table 2 PFE results of Samples A to D against $0.1 \mu \mathrm{m}$ PSL particles.

\begin{tabular}{lll}
\hline Sample & PFE & Mean \pm Standard deviation \\
\hline Sample A (Test 1) & $16.2 \%$ & $22.9 \% \pm 6.0 \%$ \\
Sample A (Test 2) & $24.9 \%$ & \\
Sample A (Test 3) & $27.6 \%$ & \\
Sample B (Test 1) & $57.5 \%$ & $51.6 \% \pm 5.1 \%$ \\
Sample B (Test 2) & $48.6 \%$ & \\
Sample B (Test 3) & $48.7 \%$ & \\
Sample C (Test 1) & $37.4 \%$ & $37.4 \% \pm 0.1 \%$ \\
Sample C (Test 2) & $37.3 \%$ & \\
Sample C (Test 3) & $42.4 \%$ & \\
Sample D (Test 1) & $86.3 \%$ & $88.3 \% \pm 1.8 \%$ \\
Sample D (Test 2) & $89.7 \%$ & \\
Sample D (Test 3) & $89.0 \%$ & \\
\hline
\end{tabular}

the lack of a filter layer between the outer and inner layers, which suggests the importance of a filter layer (O'Kelly et al., 2020). This also implies that regular fabrics such as woven fabric (W100) and knitted fabric (J92) cannot effectively block ultrafine particles. Secondly, the better performance of Sample B as opposed to Sample C indicates that the weight of the filter layer has an effect on the PFE. The filter used for Samples B and C is a non-woven interlining. The fibres are randomly laid out on the surface and bonded by using adhesion or heat. It is not surprising that a heavier filter with the same surface area has a denser and tighter structure, which is more beneficial for particle filtration. Thirdly, the much higher PFE of Sample D as opposed to Sample C is related to the material of the filter layer, which shows its key role in manufacturing a mask with a high PFE (Satish et al., 2017). The PTFE membrane used in Sample D has a denser structure than the PP membrane used in Sample C.

In order to better understand the role of the different materials used in masks to determine the PFE, their morphology was examined by using a scanning electron microscope (SEM). Fig. 4 shows the SEM images of the W100, J92, non-woven PP fabric and PTFE membrane. Clearly, the structure of W100 and J92 is neat and orderly, while that of the non-woven PP fabric and PTFE membrane is criss-crossing and more complex. Specifically, while the pore size of the non-woven PP fabric is comparable to that of the W100 and J92 fabrics, its random fibre or criss-cross structure provides many tiny voids that trap particles, thereby promoting the filtration of ultrafine particles. A multilayer structure can be observed for the PTFE membrane. In addition to the same criss-cross structured layer as the bottom of the non-woven PP sample, the surface of the PTFE membrane has a netlike structure. This netlike structure is composed of millions of ultrafine fibres which impart a "shielding effect" on the top, and thus greatly promotes particle filtration (Matulevicius et al., 2014). The multilayer structure effectively prevents the penetration of particles, thus resulting in a high PFE.

\section{Reusable Masks}

Mask reusability was determined based on the PFE 

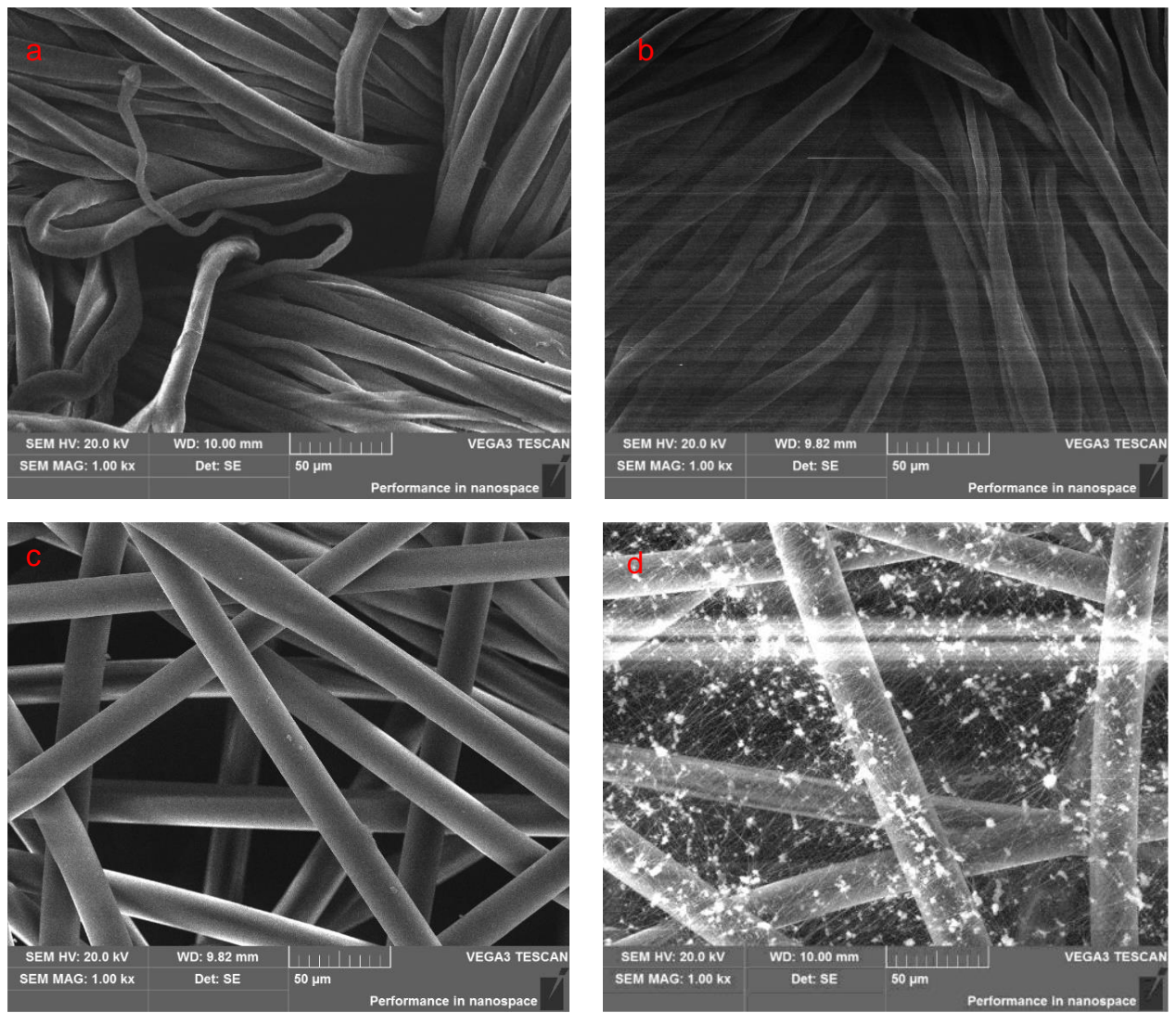

Fig. 4. Morphology of samples prior to washing. (a) W100; (b) J92; (c) non-woven PP fabric; and (d) PTFE membrane.

against PSL particles that are $0.1 \mu \mathrm{m}$ in size after the mask was washed. The washing process was gentle with no squeezing. The samples were then air dried. Since Sample D (with a PTFE membrane as the filter layer) has the best performance in filtering ultrafine particles among the four developed masks in this study, it was selected to further examine its reusability after 30 washings. For comparison purposes, the PFE of a disposable mask (i.e., the surgical mask) after one washing and Sample B (with a non-woven PP material as the filter layer) after 30 washings was measured. Table 3 presents the tested PFE values of the washed surgical mask, and Samples B and D. It can be observed that the PFE of the surgical mask is significantly reduced from $80.2 \% \pm 7.6 \%$ to $19.8 \% \pm 2.4 \%$ after one washing $(p<0.05)$. However, no significant reduction in the PFE is observed for Sample B after 30 washings. The PFE of Sample B is reduced from $51.6 \% \pm 5.1 \%$ to $43.4 \% \pm 7.9 \%$ ( $p>0.05)$. In contrast, the reduction is indeed significant in the PFE for Sample D after 30 washings ( $p<0.05$ ), which changes from $88.3 \% \pm 1.8 \%$ to $72.0 \% \pm 2.5 \%$. It is worth noting that the PFE of Sample D after 30 washings is still comparable to that of the unwashed surgical mask $(p>0.1)$, which implies that Sample D is highly effective against ultrafine particles even after multiple washings and is therefore reusable. Noteworthily, $p>0.1$ is typically trichotomized into not statistically significant at conventional levels (Gelman, 2012; Figueiredo Filho et al., 2013; Brandt-Kobele, 2014).

Fig. 5 presents the SEM images of W100, J92, non-woven PP layer and PTFE membrane after 30 washings. Some damage (red circled areas) can be observed in W100 and J92, while the non-woven PP layer and PTFE membrane can almost retain the same structure as that before washing (Fig. 4). As discussed in Section 3.2, the filter layer is critical to the PFE of a mask. Therefore, even though the outer and inner layers of Samples B and D are slightly damaged, the PFE values of Samples B and D do not decrease significantly, due to the intactness of the filter layer after the washing process. Overall, it is impractical to reuse surgical masks because after only one washing, the surgical mask is no longer effective in filtering particles (PFE < 20\%). Instead, Sample D can be reused at least 30 times as the material retains a high PFE $(>70 \%)$. The intactness of the filter layer is paramount to the reusability of a mask. Therefore, using a PTFE membrane as the filter layer of masks would provide optimal reusable masks.

\section{Air Permeability}

Table 4 shows the air permeability of N95, KF94, surgical and self-developed masks. For the commercial masks, surgical mask is the most breathable with an air permeability of $0.64 \pm 0.02 \mathrm{kPa} \cdot \mathrm{s} \mathrm{m}^{-1}$, followed by the N95 mask $(1.06 \pm$ $\left.0.04 \mathrm{kPa} \cdot \mathrm{s} \mathrm{m}^{-1}\right)$ and the KF94 mask $\left(1.39 \pm 0.05 \mathrm{kPa} \cdot \mathrm{s} \mathrm{m}^{-1}\right)$. The results are analogous to a recent research report, indicating the accuracy of the results in this study (Suen et al., 2020). As for the self-developed masks, the air permeability is $1.41 \pm$ $0.04 \mathrm{kPa} \cdot \mathrm{s} \mathrm{m}^{-1}, 1.61 \pm 0.05 \mathrm{kPa} \cdot \mathrm{s} \mathrm{m}^{-1}, 1.55 \pm 0.04 \mathrm{kPa} \cdot \mathrm{s} \mathrm{m}^{-1}$ and $1.93 \pm 0.08 \mathrm{kPa} \cdot \mathrm{s} \mathrm{m}^{-1}$ for Samples A-D, respectively. The air permeability is anti-correlated with the PFE for the 
Table 3. PFE of surgical and reusable masks after washing.

\begin{tabular}{lll}
\hline Sample & PFE & Mean \pm Standard deviation \\
\hline Surgical mask - 1 wash (Test 1) & $17.2 \%$ & $19.8 \% \pm 2.4 \%$ \\
Surgical mask - 1 wash (Test 2) & $20.4 \%$ & \\
Surgical mask - 1 wash (Test 3) & $21.9 \%$ & \\
Sample B - washed 30 times (Test 1) & $52.4 \%$ & $43.4 \% \pm 7.9 \%$ \\
Sample B - washed 30 times (Test 2) & $40.2 \%$ & \\
Sample B - washed 30 times (Test 3) & $37.5 \%$ & $72.0 \% \pm 2.5 \%$ \\
Sample D - washed 30 times (Test 1) & $70.2 \%$ & \\
Sample D - washed 30 times (Test 2) & $71.0 \%$ & \\
Sample D - washed 30 times (Test 3) & $74.9 \%$ & \\
\hline
\end{tabular}
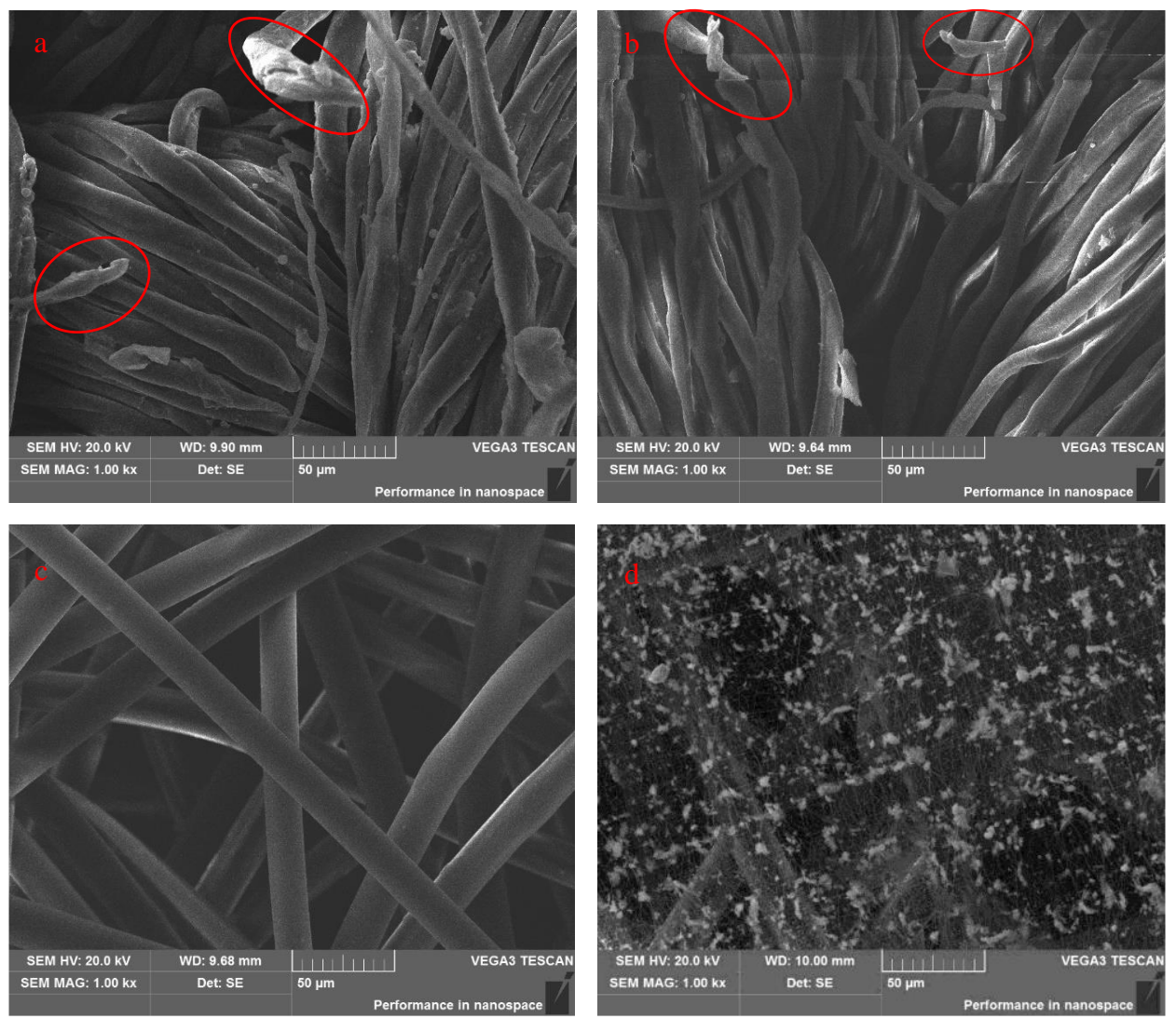

Fig. 5. Morphology of samples after 30 washings: (a) W100; (b) J92; (c) PP membrane; and (d) PTFE membrane

Table 4. Air permeability of N95, KF94, surgical and selfdeveloped masks.

\begin{tabular}{ll}
\hline Sample & Air permeability $\left(\mathrm{kPa} \cdot \mathrm{s} \mathrm{m}^{-1}\right)$ \\
\hline Surgical mask & $0.64 \pm 0.02$ \\
N95 mask & $1.06 \pm 0.04$ \\
KF94 mask & $1.39 \pm 0.05$ \\
Sample A & $1.41 \pm 0.04$ \\
Sample B & $1.61 \pm 0.05$ \\
Sample C & $1.55 \pm 0.04$ \\
Sample D & $1.93 \pm 0.08$ \\
\hline
\end{tabular}

self-developed masks. Although a multi-layer structure and/or dense filter can lead to higher PFE, the breathability of the mask may be poor. It is therefore essential for masks to achieve high PFE with acceptable air permeability (Li et al., 2006). Compared to the commercial masks, the selfdeveloped masks have lower air permeability $(p<0.05)$, except for Sample A versus KF94 mask ( $p>0.05)$. The use of woven fabrics as the inner and outer layers in the selfdeveloped masks is likely the main cause for the poorer breathability (Konda et al., 2020). Nevertheless, the selfdeveloped masks still possess relatively high permeability (i.e., $<2 \mathrm{kPa} \cdot \mathrm{s} \mathrm{m}^{-1}$ ), proving the availability of the selfdeveloped masks (Xu et al., 2020).

\section{CONCLUSIONS}

In the study, a testing system of the PFE of face masks has been developed based on standardized methods. The system is validated by measuring the PFE of three commercial masks (i.e., KF94, N95 and surgical masks) against standard 
PSL particles that are $0.1 \mu \mathrm{m}$ in size and $\mathrm{NaCl}$ particles that range from 0.01-1.0 $\mu \mathrm{m}$. The comparable PFE of the N95, KF94 and surgical masks found here against the values reported in previous studies validate the reliability of our testing system. Afterwards, different fabrics are tested to produce reusable masks, and their PFE is also measured with the proposed testing system. The results indicate that a filter layer is vital for a mask to have a high PFE. Heavier filters with the same surface area as that of the lighter filters contribute to increase of the PFE. Among the four developed masks in this study, the sample with a PTFE membrane (Sample D) is the most effective, with a PFE of $88.3 \% \pm$ $1.8 \%$, which is mainly due to the dense and multilayer structure of the PTFE membrane. The permeability of the self-developed masks is between 1.41 and $1.93 \mathrm{kPa} \cdot \mathrm{s} \mathrm{m}^{-1}$, which is not as breathable as the commercial masks. After 30 washes, the PFE of Sample D is slightly reduced to $72.0 \%$ $\pm 2.5 \%$, thus indicating its potential reusability. The reusability is mainly attributed to the intactness of the filter layer during the washing process.

\section{ACKNOWLEDGEMENTS}

This study was supported by the University Strategic Importance Scheme of Hong Kong Polytechnic University (1-ZE1M), and the Innovation and Technology Commission of Hong Kong Special Administrative Region under the Public Sector Trial Scheme for the Prevention and Control of COVID-19 in Hong Kong (SST/029/20GP). We would like to express our heartfelt thanks to Professor Ping-kong Wai and Professor Hau-chung Man for their coordination efforts.

\section{SUPPLEMENTARY MATERIAL}

Supplementary data associated with this article can be found in the online version at https://doi.org/10.4209/aaqr.2 020.06.0275

\section{REFERENCES}

Amendola, L., Saurini, M., Di Girolamo, F. and Arduini, F. (2020). A rapid screening method for testing the efficiency of masks in breaking down aerosols. Microchem. J. 157: 104928. https://doi.org/10.1016/j.mic roc. 2020.104928

Bałazy, A., Toivola, M., Adhikari, A., Sivasubramani, S. K., Reponen, T. and Grinshpun, S.A. (2006). Do N95 respirators provide $95 \%$ protection level against airborne viruses, and how adequate are surgical masks? Am. J. Infect. Control 34: 51-57. https://doi.org/10.1016/j.ajic.2 005.08.018

Brandt-Kobele, O.C. (2014). Comprehension of verb inflection in German-speaking children (Vol. 6). Universitätsverlag Potsdam.

Cheng, S.T. (2020). Covid-19: are face masks a good longterm strategy? BMJ 369: m2005. https://doi.org/10.1136/ bmj.m2005

Figueiredo Filho, D.B., Paranhos, R., Rocha, E.C.D.,
Batista, M., Silva Jr, J.A.D., Santos, M.L.W.D. and Marino, J.G. (2013). When is statistical significance not significant? Braz. Political Sci. Rev. 7: 31-55. https://doi.org/10.1590/S1981-38212013000100002

Fukasawa, T. and Adachi, Y. (2010). Direct observation on the Brownian coagulation of PSL particles through optical microscope in the regime near critical coagulation concentration (CCC). J. Colloid Interface Sci. 344: 343347. https://doi.org/10.1016/j.jcis.2009.12.066

Gelman, A. (2012, September 26). What do statistical pvalues mean when the sample $=$ the population. Statistical Modeling, Causal Inference, and Social Science. https://statmodeling.stat.columbia.edu/2012/09/26/what-dostatistical-p-values-mean-when-the-sample-the-population/

Kohli, R. and Mittal, K.L. (2011). Developments in surface contamination and cleaning, Volume 4: Detection, characterization, and analysis of contaminants. William Andrew.

Konda, A., Prakash, A., Moss, G.A., Schmoldt, M., Grant, G.D. and Guha, S. (2020). Aerosol filtration efficiency of common fabrics used in respiratory cloth masks. ACS Nano 14: 6339-6347. https://doi.org/10.1021/acsnano.0c 03252

Lam, S.C., Suen, L.K.P. and Cheung, T.C.C. (2020). Global risk to the community and clinical setting: Flocking of fake masks and protective gears during the COVID-19 pandemic. Am. J. Infect. Control 48: 964-965. https://doi.org/10.1016/j.ajic.2020.05.008

Li, Y., Wong, T., Chung, A.J., Guo, Y.P., Hu, J.Y., Guan, Y.T. and Newton, E. (2006). In vivo protective performance of N95 respirator and surgical facemask. Am. J. Ind. Med. 49: 1056-1065. https://doi.org/10.1002/ajim.20395

Liu, H.L. and Hsu, C.M. (2003). The effectiveness of different levels of face masks is very different. (in Chinese) https://scitechvista.nat.gov.tw/c/s9p2.htm

Liu, L. (2020). Emerging study on the transmission of the Novel Coronavirus (COVID-19) from urban perspective: Evidence from China. Cities 103: 102759. https://doi.org/10.1016/j.cities.2020.102759

MacIntyre, C.R. and Chughtai, A.A. (2020). A rapid systematic review of the efficacy of face masks and respirators against coronaviruses and other respiratory transmissible viruses for the community, healthcare workers and sick patients. Int. J. Nurs. Stud. 108: 103629. https://doi.org/10.1016/j.ijnurstu.2020.103629

Matulevicius, J., Kliucininkas, L., Martuzevicius, D., Krugly, E., Tichonovas, M. and Baltrusaitis, J. (2014). Design and characterization of electrospun polyamide nanofiber media for air filtration applications. J. Nanomater. 2014: 859656. https://doi.org/10.1155/2014/859656

Ningthoujam, R. (2020). COVID 19 can spread through breathing, talking, study estimates. Curr. Med. Res. Pract. 10: 132-133. https://doi.org/10.1016/j.cmrp.2020.05.003

O'Kelly, E., Pirog, S., Ward, J. and Clarkson, P.J. (2020). Ability of fabric facemasks materials to filter ultrafine particles at coughing velocity-for home made and fabric face mask creation. BMJ Open 10: e039424 https://doi.org/10.1136/bmjopen-2020-039424

Rengasamy, S., Miller, A. and Eimer, B.C. (2011). Evaluation 
of the filtration performance of NIOSH-approved N95 filtering facepiece respirators by photometric and number-based test methods. J. Occup. Environ. Hyg. 8: 23-30. https://doi.org/10.1080/15459624.2010.515556

Rengasamy, S., Shaffer, R., Williams, B. and Smit, S. (2017). A comparison of facemask and respirator filtration test methods. J. Occup. Environ. Hyg. 14: 92-103. https://doi.org/10.1080/15459624.2016.1225157

Sachinidou, P., Bahk, Y.K. and Wang, J. (2017). An integrative model for the filtration efficiencies in realistic tests with consideration of the filtration velocity profile and challenging particle size distribution. Aerosol Sci. Technol. 51: 178-187. https://doi.org/10.1080/02786826. 2016.1254720

Satish, S., Swanson, J.J., Xiao, K., Viner, A.S., Kittelson, D.B. and Pui, D.Y. (2017). Gravimetric measurements of filtering facepiece respirators challenged with diesel exhaust. Ann. Work Expo. Health 61: 737-747. https://doi.org/10.1093/annweh/wxx044

Suen, L.K.P., Guo, Y.P., Ho, S.S.K., Au-Yeung, C.H. and Lam, S.C. (2020). Comparing mask fit and usability of traditional and nanofibre N95 filtering facepiece respirators before and after nursing procedures. J. Hosp. Infect. 104: 336-343. https://doi.org/10.1016/j.jhin.2019.09.014

Tomar, A. and Gupta, N. (2020). Prediction for the spread of COVID-19 in India and effectiveness of preventive measures. Sci. Total Environ. 728: 138762. https://doi.org/10.1016/j.scitotenv.2020.138762

Wang, J. and Tronville, P. (2014). Toward standardized test methods to determine the effectiveness of filtration media against airborne nanoparticles. J. Nanopart. Res. 16: 2417. https://doi.org/10.1007/s11051-014-2417-Z

Xu, Q., Liu, H., Zhong, X., Jiang, B. and Ma, Z. J. (2020). Permeable weldable elastic fiber conductor for wearable electronics. ACS Appl. Mater. Interfaces 12: 36609 36619. https://doi.org/10.1021/acsami.0c08939

Yang, S., Lee, W.M.G., Huang, H.L., Huang, Y.C., Luo, C.H., Wu, C.C. and Yu, K.P. (2007). Aerosol penetration properties of an electret filter with submicron aerosols with various operating factors. J. Environ. Sci. Health., Part A 42: 51-57. https://doi.org/10.1080/109345206010 15651

Yasui, H. and Higashitani, K. (1988). Seed coagulation of latex particles. J. Colloid Interface Sci. 125: 472-477. https://doi.org/10.1016/0021-9797(88)90011-2

Received for review, June 1, 2020 Revised, August 9, 2020 Accepted, September 9, 2020 